\title{
Moving breather collisions in the Peyrard-Bishop DNA model
}

\author{
A Alvarez ${ }^{1}$, FR Romero ${ }^{1}, \mathrm{~J} \mathrm{Cuevas}^{2}$, and JFR Archilla ${ }^{2}$ \\ 1 Grupo de Física No Lineal. Área de Física Teórica. Facultad de Física. Universidad \\ de Sevilla. Avda. Reina Mercedes, s/n. 41012-Sevilla (Spain), \\ azucena@us.es, \\ WWW home page: http://www.grupo.us.es/gfnl \\ 2 Grupo de Física No Lineal. Departamento de Fisica Aplicada I. ETSI Informática. \\ Universidad de Sevilla. Avda. Reina Mercedes, s/n. 41012-Sevilla (Spain)
}

\begin{abstract}
We consider collisions of moving breathers (MBs) in the Peyrard-Bishop DNA model. Two identical stationary breathers, separated by a fixed number of pair-bases, are perturbed and begin to move approaching to each other with the same module of velocity. The outcome is strongly dependent of both the velocity of the MBs and the number of pair-bases that initially separates the stationary breathers. Some collisions result in the generation of a new stationary trapped breather of larger energy. Other collisions result in the generation of two new MBs. In the DNA molecule, the trapping phenomenon could be part of the complex mechanisms involved in the initiation of the transcription processes.
\end{abstract}

Key words: Discrete breathers, intrinsic localized modes, moving breathers, breather collisions, Peyrard-Bishop model.

\section{Introduction and model set-up}

The DNA molecule is a discrete system consisting of many atoms having a quasione-dimensional structure. It can be considered as a complex dynamical system, and, in order to investigate some aspects of the dynamics and the thermodynamics of DNA, several mathematical models have been proposed. Among them, it is worth remarking the Peyrard-Bishop model [1] introduced for the study of DNA thermal denaturation. This model, as well as some variations of it, have also been used extensively for the study of some dynamical properties of DNA.

The study of discrete breathers (DBs) in chains of oscillators is an active research field in nonlinear physics $[2,3,4,5]$. Under certain conditions, stationary breathers can be put in motion if they experience appropriate perturbations [6], and they are called moving breathers (MBs). There are no exact solutions for MBs, but they can be obtained by means of numerical calculations.

In the Peyrard-Bishop model, the existence of DBs has been demonstrated [7, 8], and DBs are thought to be the precursors of the bubbles that appear prior to the transcription processes in which large fluctuations of energy have been 
experimentally observed. Some studies about the existence and properties of MBs in the Peyrard-Bishop model including dipole-dipole dispersive interaction are carried out in $[9,10]$.

In this work, we consider the Peyrard-Bishop DNA model, which Hamiltonian can be written as

$$
H=\sum_{n=1}^{N}\left(\frac{1}{2} m \dot{u}_{n}^{2}+D\left(e^{-b u_{n}}-1\right)^{2}+\frac{1}{2} \varepsilon_{0}\left(u_{n+1}-u_{n}\right)^{2}\right],
$$

the term $\frac{1}{2} m \dot{u}_{n}^{2}$ represents the kinetic energy of the nucleotide of mass $m$ at the $n^{\text {th }}$ site of the chain, and $u_{n}$ is the variable representing the transverse stretching of the hydrogen bond connecting the base at the $n^{\text {th }}$ site. The Morse potential, i.e., $D\left(e^{-b u_{n}}-1\right)^{2}$, represents the interaction energy due to the hydrogen bonds within the base pairs, being $D$ the well depth, which corresponds to the dissociation energy of a base pair, and $b^{-1}$ is related to the width of the well. The stacking energy is $\frac{1}{2} \varepsilon_{0}\left(u_{n+1}-u_{n}\right)^{2}$, where $\varepsilon_{0}$ is the stacking coupling constant.

In scaled variables this Hamiltonian can be writing as:

$$
H=\sum_{n}\left[\frac{1}{2} \dot{u}_{n}^{2}+V\left(u_{n}\right)+\frac{1}{2} \varepsilon\left(u_{n}-u_{n+1}\right)^{2}\right],
$$

where $u_{n}$ represents the displacement of the $n^{t h}$ pair-base from the equilibrium position, $\varepsilon$ is the coupling parameter and $V\left(u_{n}\right)$ is:

$$
V\left(u_{n}\right)=\frac{1}{2}\left(\exp \left(-u_{n}\right)-1\right)^{2} .
$$

Time-reversible, stationary breathers can be obtained using methods based on the anti-continuous limit [11]. At $t=0, \dot{u}_{n}=0, \forall n$, and the displacements of a breather centered at $n_{0}$ are denoted by $\left\{u_{S B, n}\right\}$. A moving breather $\left\{u_{t, n}\right\}$ can be obtained with the following initial displacements and velocities:

$$
\begin{aligned}
& u_{M B, n}^{0}=u_{S B, n} \cos \left(\alpha\left(n-n_{0}\right)\right) \\
& \dot{u}_{M B, n}^{0}= \pm u_{S B, n} \sin \left(\alpha\left(n-n_{0}\right)\right) .
\end{aligned}
$$

The plus-sign corresponds to a breather moving towards the positive direction and the minus one, the opposite. This procedure works as well as the marginalmode method [6] and gives good mobility for a large range of $\varepsilon$. The translational velocity and the translational kinetic energy of the MB increase with $\alpha$. We use Eqs. (4) as initial conditions to integrate the dynamical equations using a symplectic algorithm [13].

The study begins generating two identical stationary breathers, with the same frequency, separated by a fixed number of pair-bases between their centers. We call $N_{c}$ the number of pair-bases separating initially the centers of the two DBs. Both breathers are in phase, that is, before the perturbation, each breather is 
always like the mirror image of the other one. The perturbation should be given simultaneously to both breathers and the initial conditions of each breather given by Eqs. (4), with the plus sign for one breather and the minus sign for the other

one. In this way the MBs travel with the same modulus of velocity, but opposite directions, and they are in phase.

\section{Results and conclusions}

We can analyze collisions with a fixed value of the parameter $\alpha$ and different values of $N_{c}$ so that the colliding MBs keep unchanged. Also, we can analyze collisions varying the parameter $\alpha$ maintaining fixed the number $N_{c}$, thus the colliding MBs change for each value of $\alpha$. We write

$$
N_{c}=N_{o}+j j,
$$

where $N_{o}$ is a fixed number to guarantee that the breathers are initially far apart, and $j j$ is a positive even number.

In the first approach we fix the parameter $\alpha$ and perturb the DBs varying their separation $N_{c}$, thus the only difference between two collisions is the time passed between the initial perturbation and the initiation of the collision.

We consider collisions where the DBs are in phase and perturbed simultaneously. We have taken $N_{o}=40$ and $j j$ varies in the interval $[0,100]$ with step size 2 . Then, up to fifty different collisions can be analyzed for a fixed value of $\alpha$ and $\varepsilon$.

We have performed an extensive numerical simulations considering different values of the coupling parameter $\varepsilon$, and MBs with different values of the wave number $\alpha$. The values of $\varepsilon$ have been taken in the interval $[0.13,0.35]$ with step size 0.01 . For each value of $\varepsilon$ the values of $\alpha$ have been taken in the interval $[0.030,0.200]$ with step size 0.002 . We present the results obtained with $\varepsilon=0.32$ and $\alpha=0.048 ; \alpha=0.138 ; \alpha=0.18$, which correspond to MBs with increasing velocities. These values are representative of the different scenarios that can be found. Fig. 1 represents the trapped energy versus $j j$ for these three cases. The qualitative results are similar for other values of the parameters $(\varepsilon, \alpha)$.

Fig. 1(left) corresponds to the case with the smallest velocity, i.e., $\alpha=0.048$, the distribution of points appears in a narrow band and there are no points with trapped energy close to zero. When the MBs have small enough kinetic energy, most of the energy gets trapped after the collision and two small MBs are generated traveling with opposite directions, they transport the remaining energy except a small part that is lost in the form of phonon radiation. Notice that for $j j$ up to 30, the points oscillate following a repetitive regular pattern, and this regularity begin to change as $j j$ increases.

For intermediate values of $\alpha$ the phenomenon of non-trapping, or breather generation, appears for some values of $j j$. This can be appreciated in Fig. 1(central), obtained with $\alpha=0.138$, where some points appear with trapped energy close to zero, this means that after the collision almost all the energy is transported 
by two emerging MBs with the same velocities that the incoming MBs'. For this value of $\alpha$ two points in the upper band is followed by one point that fall down close to zero.

For $\alpha=0.18$, the upper band is divided in pieces and another fragmented lower band appears. There are alternating intervals of $N_{c}$ values corresponding to the upper band and other corresponding to the lower band. This means that there are some successive values of $j j$ associated with trapping, followed by other ones associated with breather generation, see Fig. 1(right).
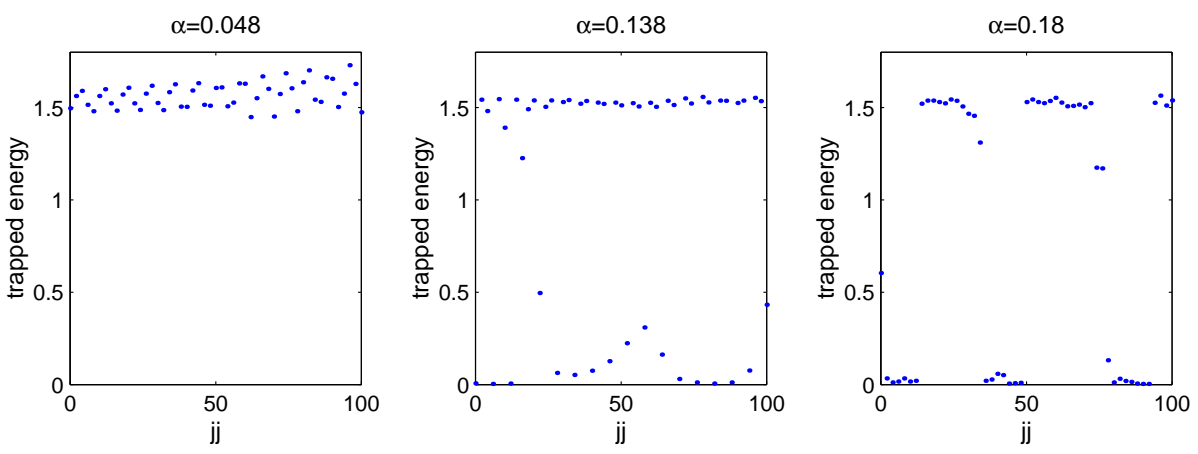

Fig. 1. Three distributions of points representing the trapped energy versus $j j$, for $\alpha=0.048, \alpha=0.138$, and $\alpha=0.18$, respectively. Coupling parameter $\varepsilon=0.32$ and breather frequency $\omega_{\mathrm{b}}=0.8$.

Fig. 2 shows the displacements versus time for eight collisions of Fig. 1(central), corresponding to $j j=34, \ldots ., 48$, with the fixed value $\alpha=0.138, \varepsilon=0.32$ and breather frequency $\omega_{\mathrm{b}}=0.8$.

Fig. 3 shows the evolution of the trapped energy for the collisions with $j j=$ $34, \ldots 40$ of Fig. 2. For $j j=34$ and $j j=40$ two new breathers are generated. The other cases correspond to breather trapping with breather generation.

It is interesting to study the collisions maintaining fixed the number $N_{c}$ and varying $\alpha$ for fixed values of $\omega_{\mathrm{b}}$ and $\varepsilon$. In real DNA the MBs could be generated at fixed points of the chain by the action of proteins. Obviously, the phenomenology is similar to the previous case and the study has permitted to observe a great sensitivity of the outcomes with respect to the parameter $\alpha$ (Ref.[14]). To see this, let us consider the results for three nearness values of $\alpha$ with $N_{c}=40$, $\varepsilon=0.32$ and $\omega_{\mathrm{b}}=0.8$ :

For $\alpha=0.1370$, the collision produces three new breathers, a trapped breather containing most of the initial energy and two new MBs.

For $\alpha=0.1372$, there is a noticeable attenuation of the amplitude of the trapped breather, which anticipates an entirely new outcome. The emerging MBs contain most of the initial energy.

For $\alpha=0.1374$, there is no trapping and two new MBs emerge with almost the same velocity that the colliding breathers'. 

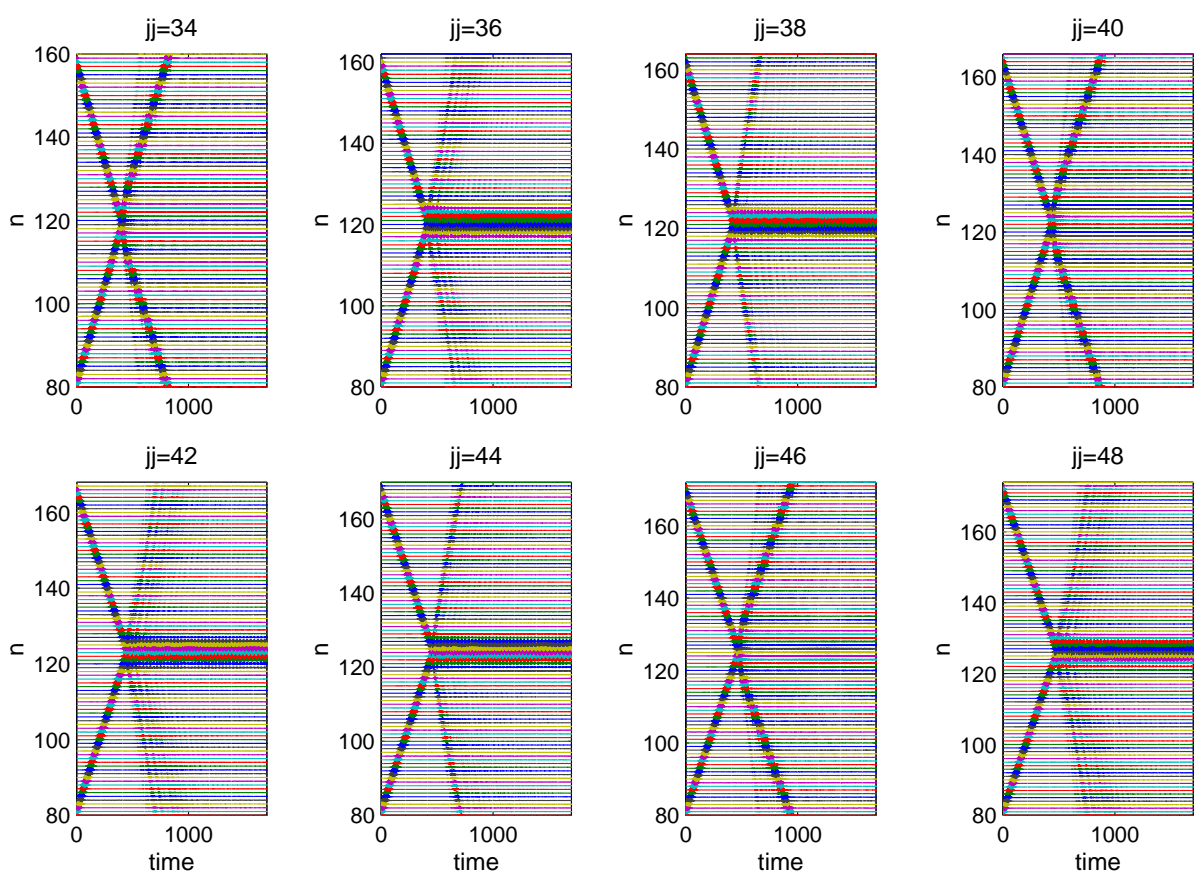

Fig. 2. Displacements versus time for eight collisions corresponding to $j j=34, \ldots, 48$, with the fixed value $\alpha=0.138$. Coupling parameter $\varepsilon=0.32$ and breather frequency $\omega_{\mathrm{b}}=0.8$.
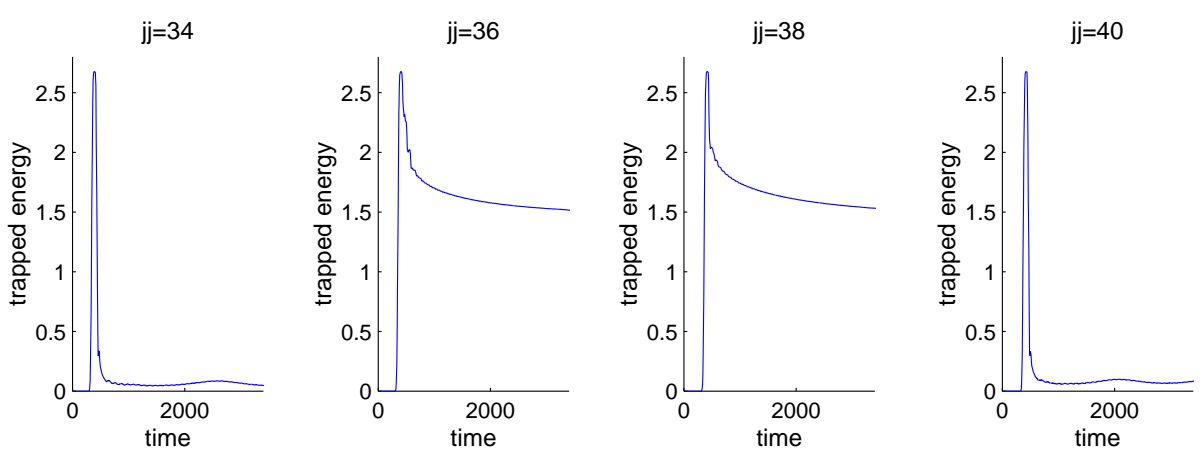

Fig. 3. Trapped energy versus time corresponding to the first four collisions of Fig. 2, respectively. 
The previous studies let us to conclude that for a given values of $\varepsilon$ and $\omega_{\mathrm{b}}$, the relevant parameters to determine the outcomes of the collisions are both $\alpha$ and the number $N_{c}$.

The simulations of MB collisions in the Peyrard-Bishop DNA model show a new mechanism for concentrating energy in DNA. When two MBs collide, it is possible, in some favorable cases, to get stationary trapped breathers with more energy than the colliding breathers. These breathers are also movable and after colliding with other ones, could give rise to even more energetic stationary breathers. This mechanism could be part of the complex mechanisms involved in the initiation of the transcription processes.

We are performing extensive numerical simulations of other types of collisions that can appear in the DNA molecule, which will be published elsewhere.

\section{References}

1. Peyrard, M., Bishop, A.R.: Statistical mechanics of a nonlinear model for DNA denaturation. Phys. Rev. Lett. 62, 2755-2758 (1989)

2. Dauxois, T., Mackay, R.S., Tsironis, G.P.: Nonlinear Physics: Condensed Matter, Dynamical Systems and Biophysics - A Special Issue dedicated to Serge Aubry. Physica D. 216, 1-246 (2006)

3. Kivshar, Yu.S., Flach, S.: Nonlinear localized modes: physics and applications. Chaos 13, 586-799 (2003)

4. Flach, S., Mackay, R.S.: Localization in nonlinear lattices. Physica D. 119, 1-238 (1999)

5. Flach, S., Willis, C.R.: Discrete breathers. Phys. Rep. 295, 181-264 (1998)

6. Aubry, S., Cretegny, T.: Mobility and reactivity of discrete breathers. Physica D. 119, 34-46 (1998)

7. Dauxois, T., Peyrard, M., Willis, C.R.: Localized breather-like solution in a discrete Klein-Gordon model and application to DNA. Physica D. 57, 267-282 (1992)

8. Dauxois, T., Peyrard, M., Bishop, A.R.: Dynamics and thermodynamics of a nonlinear model for DNA denaturation. Phys. Rev. E. 47, 684-695 (1993)

9. Cuevas, J., Archilla, J.F.R., Gaididei, Yu.B., Romero, F.R.: Moving breathers in a DNA model with competing short- and long-range dispersive interactions. Physica D. 163, 106-126 (2002)

10. Alvarez, A., Romero, F.R., Archilla, J.F.R., Cuevas, J., Larsen, P.V.: Breather trapping and breather transmission in a DNA model with an interface. Eur. Phys. J. B 51, 119-130 (2006)

11. Marín, J.L., Aubry, S.: Breathers in nonlinear lattices: Numerical calculation from the anticontinuous limit. Nonlinearity 9, 1501-1528 (1996)

12. Dmitriev, S.V., Kevrekidis, P.G., Malomed, B.A., Frantzeskakis, D.J.: Two-soliton collisions in a near-integrable lattice system. Phys. Rev. E 68, 056603, 1-7 (2003)

13. Sanz-Serna, J.M., Calvo, M.P.: Numerical Hamiltonian problems. Chapman and Hall (1994)

14. Alvarez, A., Romero, F.R., Cuevas, J., Archilla, J.F.R.: Discrete moving breather collisions in a Klein-Gordon chain of oscillators. Phys. Lett. A 372, 1256-1264 (2008) 